\title{
Baseline Decrement in Patients with Mild Myasthenia Gravis Predicts Immunomodulation Treatment
}

\author{
Alon Abraham (1D, Leif E. Lovblom, Vera Bril
}

\begin{abstract}
To explore whether higher degrees of electrophysiological abnormalities are associated with a more frequent exposure to a more aggressive treatment regimen, we performed a retrospective chart review of patients attending the neuromuscular clinic from June 2012 to December 2015 and included 87 patients. We compared treatment regimens during the follow-up period between patients with high and low jitter and decrement. Myasthenia gravis patients with high jitter or decrement at baseline were more frequently treated with intravenous immunoglobulins (IVIG) and/or plasma exchange (PLEX) during the follow-up period. In patients with mild disease, IVIG or PLEX treatment was associated with high decrement.
\end{abstract}

RÉSUMÉ: La diminution initiale du potentiel moteur de patients atteints de myasthénie grave peut permettre de prédire le type d'immunomodulation thérapeutique prodiguée. Afin d'explorer dans quelle mesure des niveaux plus élevés d'anomalies électro-physiologiques peuvent être associés à une exposition davantage fréquente à des régimes de traitement plus vigoureux, nous avons effectué un examen rétrospectif des dossiers de patients, 87 au total, s'étant présentés à une clinique neuromusculaire de juin 2012 à décembre 2015. Nous avons alors comparé les régimes de traitement des patients montrant de basses mesures de gigue (jitter) et une faible diminution d'amplitude du potentiel d'action au cours de leur période de suivi avec les régimes de traitement d'autres patients pour qui ces mesures étaient élevées. Les patients atteints de myasthénie grave (MG) dont les mesures de gigue et la diminution d'amplitude du potentiel d'action étaient initialement élevées ont été plus fréquemment traités, lors d'un suivi, avec des immunoglobulines intraveineuses et/ou des échanges plasmatiques. Chez les patients atteints de la forme bénigne de cette maladie, ces deux traitements ont été associés à une diminution d'amplitude du potentiel d'action plus élevée.

Keywords: Myasthenia gravis, Repetitive nerve stimulation, Single fiber EMG, QMGS, Disease severity, Treatment

doi:10.1017/cjn.2019.246

Can J Neurol Sci. 2019; 46: 762-766

Electrophysiological confirmation of impaired neuromuscular transmission is commonly performed in myasthenia gravis (MG) and includes single fiber electromyography (SFEMG) and repetitive nerve stimulation (RNS). While decrement reflects neuromuscular transmission failure at the neuromuscular junction, SFEMG can detect even a delay in neuromuscular transmission and is therefore more sensitive. The value of electrophysiological evaluation in MG extends beyond the ability to diagnose impaired neuromuscular transmission and is correlated with disease severity. ${ }^{1,2}$ In addition, the electrophysiological findings might assist in predicting the risk for disease exacerbations and even suggest treatment responsiveness. ${ }^{3}$ Similar results were not found for acetylcholine receptor antibodies. ${ }^{4}$ In the current study, we aimed to explore whether higher degrees of electrophysiological abnormality at baseline are associated with more frequent exposure to a more aggressive treatment regimen. As electrophysiological findings are associated with worse clinical severity ${ }^{2}$ and a higher risk for exacerbations even with mild symptoms, ${ }^{3}$ we hypothesized that more severe electrophysiological findings would be associated with more frequent exposure to more aggressive treatment regimens.

We performed a retrospective chart review of 134 patients referred to the Prosserman Family Neuromuscular Clinic at the University Health Network from June 2012 to December 2015 for possible MG, who were then diagnosed as having the disorder at this initial visit. MG diagnosis was performed by a neuromuscular expert (VB) in all patients, based on a compatible clinical presentation supported by abnormal SFEMG findings. In addition, all patients underwent routine detailed clinical history taking and comprehensive neurological examination, with assessment of disease severity by the quantitative myasthenia gravis score $(\mathrm{QMGS})^{5}$ at baseline visit in most patients. Eighty-seven patients who returned for follow-up in our clinic were included in the current study. The mean follow-up period was $2.6 \pm 1.7$ years. The remaining 47 patients who did not return for follow-up (reason unknown) did not differ from the 87 patients who did return for follow-up in terms of demographic, clinical, and

From the Neuromuscular Diseases Unit of the Department of Neurology, Tel Aviv Sourasky Medical Center, the Sackler Faculty of Medicine, Tel Aviv University, Tel Aviv, Israel (AA); Ellen and Martin Prosserman Centre for Neuromuscular Diseases, Division of Neurology, Department of Medicine, University Health Network, University of Toronto, Toronto, Canada (VB); Division of Endocrinology and Metabolism, Department of Medicine, Sinai Health System, and Lunenfeld Tanenbaum Research Institute, University of Toronto, Toronto, Canada (LEL); Institute for Research and Medical Consultations, Imam Abdulrahman Bin Faisal University, Dammam, Saudi Arabia (VB)

Received February 28, 2019. Final Revisions Submitted July 13, 2019. Date of ACCEPTANCE July 16, 2019.

Correspondence to: Alon Abraham, Neuromuscular Diseases Unit of the Department of Neurology, Tel Aviv Medical Center, the Sackler Faculty of Medicine, Tel Aviv University, 6 Weizmann St, Tel Aviv, Israel. Email: alonabmail@gmail.com 
Table 1: Comparison of baseline characteristics and treatment during follow-up period between patients with high and low decrement and jitter values

\begin{tabular}{|c|c|c|c|c|c|c|}
\hline \multirow[b]{3}{*}{$n$} & \multicolumn{3}{|c|}{ Decrement } & \multicolumn{3}{|c|}{ Jitter } \\
\hline & $<20 \%$ & $\geq \mathbf{2 0 \%}$ & $p$-value & $<\mathbf{1 0 0 \mu \mathrm { s }}$ & $\geq \mathbf{1 0 0 \mu \mathrm { s }}$ & $p$-value \\
\hline & 75 & 10 & & 48 & 39 & \\
\hline Age (y) & $59 \pm 17$ & $57 \pm 16$ & 0.72 & $60 \pm 17$ & $58 \pm 16$ & 0.44 \\
\hline Woman, $n(\%)$ & $28(37)$ & $3(30)$ & 0.74 & $18(38)$ & $14(36)$ & 0.88 \\
\hline Duration (y) & $4.0 \pm 7.0$ & $4.3 \pm 7$ & 0.88 & $4.2 \pm 7.7$ & $3.7 \pm 5.4$ & 0.75 \\
\hline Generalized & $49(65)$ & $9(90)$ & 0.16 & $27(56)$ & $33(85)$ & 0.004 \\
\hline QMGS & $7.5 \pm 4.6$ & $13.9 \pm 5.9$ & $<0.001$ & $7.2 \pm 4.9$ & $9.6 \pm 5.1$ & 0.05 \\
\hline AchRAb & $28 / 40(70)$ & $7 / 8(88)$ & 0.42 & $13 / 23(57)$ & $24 / 27(89)$ & 0.001 \\
\hline F/U Duration (y) & $2.5 \pm 1.7$ & $3.0 \pm 1.0$ & 0.40 & $2.5 \pm 1.9$ & $2.7 \pm 1.2$ & 0.60 \\
\hline Thymectomy & $20(27)$ & $5(50)$ & 0.13 & $12(25)$ & $13(33)$ & 0.39 \\
\hline
\end{tabular}

Treatment during follow-up period

\begin{tabular}{l|c|c|c|c|c|c}
\hline Mestinon & $66(88)$ & $9(90)$ & 0.85 & $41(85)$ & $35(90)$ & 0.55 \\
\hline Prednisone & $52(69)$ & $7(70)$ & 0.97 & $31(65)$ & $30(77)$ & 0.21 \\
\hline Azathioprine & $34(45)$ & $6(60)$ & 0.38 & $20(42)$ & $20(51)$ & 0.37 \\
\hline Mycophenolate & $18(24)$ & $4(40)$ & 0.28 & $11(23)$ & 0.28 \\
\hline IVIG & $14(19)$ & $5(50)$ & $\mathbf{0 . 0 2 6}$ & $7(15)$ & $13(33)$ & $\mathbf{0 . 0 4}$ \\
\hline PLEX & $7(9)$ & $4(40)$ & $\mathbf{0 . 0 0 7}$ & $3(6)$ & $8(21)$ & 0.06 \\
\hline
\end{tabular}

$\mathrm{Y}=$ years; $\mathrm{QMGS}=$ quantitative myasthenia gravis score; $\mathrm{AchRAb}=$ elevated titer of acetylcholine receptor antibodies; $F / \mathrm{U}=$ follow-up; IVIG=intravenous immunoglobulins; PLEX=plasma exchange.

Data presented as $n(\%)$ or mean $\pm \mathrm{SD}$.

Significant $p$-values $(<0.05)$ are shown in bold.

electrophysiological findings (data not shown) and were excluded from further analysis. We recorded demographics, MG-related symptoms, QMGS, and electrophysiological findings ${ }^{6}$ at the baseline (first) visit. In addition, we recorded serology results, follow-up duration, and clinical symptoms at the last follow-up visit, and MG treatments were given within the follow-up period. Only relatively common treatments given in our clinic were recorded, including pyridostigmine, prednisone, azathioprine, mycophenolate, intravenous immunoglobulins (IVIG), and plasma exchange (PLEX). QMGS was not performed at the last follow-up visit, as our practice changed in 2015 when we started to use the myasthenia gravis impairment index. ${ }^{7}$

The electrophysiological assessment included SFEMG and RNS and was performed in the frontalis muscle in all patients prior to clinical evaluation or referral letter review, to maintain testing objectivity. However, as the clinic routine is to evaluate patients with suspected MG on specific days, the clinic staff was aware of the suspicion of MG. MG-related symptoms were compared between patients who had higher $(\geq 100 \mu \mathrm{s})$ and lower mean jitter values, and between patients who had higher $(\geq 20 \%)$ and lower decrement values at the baseline visit and at the last follow-up visit. These specific electrophysiological cut-off values were used due to their simplicity, and based on a previous study demonstrating the association of higher values with a more severe disease. $^{2}$ In addition, we compared treatment regimens given within the follow-up period between patients with higher $(\geq 100 \mu \mathrm{s})$ and lower jitter and higher $(\geq 10 \%$ and $\geq 20 \%)$ and lower decrement values (in the total cohort, in patients with QMGS $<10.5$ indicating mild disease, and in patients with QMGS > 10.5, indicating moderate to severe disease). ${ }^{8}$ The Research Ethics Board of the University Health Network approved the study protocol and waived informed consent.

Statistical analysis was performed using SAS version 9.4 (SAS Institute, Cary, North Carolina, USA). Demographic, MG-related symptoms, serologic findings, and MG treatments are presented as means and standard deviations or as numbers and percent, as appropriate, and comparison of characteristics between high and low decrement and jitter values was performed using the $t$-test or the $\chi^{2}$-test. Fisher's exact test was used in place of the $\chi^{2}$-test when expected counts were less than five. Odds ratios (ORs) (and their 95\% confidence intervals) of receiving treatment in the presence of high jitter or high decrement values (compared to those with low jitter or decrement values) were calculated using logistic regression. Significance was set at a $p$-value $<0.05$ (two-tailed).

MG patients with more severely abnormal electrophysiological parameters (decrement $\geq 20 \%$ or jitter $\geq 100 \mu$ s recorded from the frontalis muscle) at baseline were more frequently seropositive and had generalized disease, with a higher QMGS at baseline. During the follow-up period, the most common treatment included mestinon, followed by prednisone and azathioprine (Table 1). There was no difference in treatment doses between groups, except higher dose of mestinon in patients with decrement $\geq 20 \%$ (324 vs. $212 \mathrm{mg}, p=0.01$ ). Patients with 


\section{Table 2: Comparison of symptoms between MG patients with high and low decrement and jitter values at baseline and last follow-up visit}

\begin{tabular}{|c|c|c|c|c|c|c|}
\hline \multirow[b]{2}{*}{ Decrement } & \multicolumn{3}{|c|}{ Baseline } & \multicolumn{3}{|c|}{ Follow-up } \\
\hline & $<20 \%$ & $\geq 20 \%$ & $p$-value & $<20 \%$ & $\geq \mathbf{2 0 \%}$ & $p$-value \\
\hline$n$ & 75 & 10 & & 75 & 10 & \\
\hline Ptosis & $49(65)$ & $7(70)$ & 0.77 & $32(43)$ & $3(30)$ & 0.44 \\
\hline Diplopia & $44(59)$ & $6(60)$ & 0.94 & $26(35)$ & $1(10)$ & 0.16 \\
\hline Bulbar & $30(40)$ & $9(90)$ & 0.005 & $17(23)$ & $6(60)$ & 0.013 \\
\hline Respiratory & $12(17)$ & $6(60)$ & 0.002 & $8(11)$ & $3(30)$ & 0.087 \\
\hline Limb weakness & $23(31)$ & $6(60)$ & 0.066 & $16(21)$ & $2(20)$ & 0.92 \\
\hline Jitter & $<\mathbf{1 0 0} \mu \mathrm{s}$ & $\geq 100 \mu \mathrm{s}$ & $p$-value & $<100 \mu \mathrm{s}$ & $\geq 100 \mu \mathrm{s}$ & $p$-value \\
\hline$n$ & 48 & 39 & & 48 & 39 & \\
\hline Ptosis & $32(67)$ & $25(64)$ & 0.81 & $23(48)$ & $12(31)$ & 0.11 \\
\hline Diplopia & $27(56)$ & $24(62)$ & 0.62 & $18(38)$ & $9(23)$ & 0.15 \\
\hline Bulbar & $14(29)$ & $26(67)$ & $<0.001$ & $10(21)$ & $14(36)$ & 0.12 \\
\hline Respiratory & $5(11)$ & $13(35)$ & $<0.01$ & $4(8)$ & $8(21)$ & 0.10 \\
\hline Limb weakness & $12(36)$ & $17(22)$ & 0.07 & $9(19)$ & $11(28)$ & 0.30 \\
\hline
\end{tabular}

Data presented as $n(\%)$.

Significant $p$-values $(<0.05)$ are shown in bold.

higher decrement at baseline $(\geq 10 \%)$ were more frequently treated with IVIG and PLEX, while patients with higher jitter were more frequently treated with IVIG (Table 1). Patients with high decrement had more frequent bulbar and respiratory symptoms at baseline, and more frequent bulbar symptoms at followup. In contrast, patients with high jitter had more frequent bulbar and respiratory symptoms only at the baseline visit (Table 2). MG patients with more severely abnormal electrophysiological parameters (either decrement $\geq 10 \%$ or jitter $\geq 100 \mu \mathrm{s}$ ) and patients with high QMGS $(>10.5)$ were more frequently treated with IVIG and PLEX (Table 3 ). However, in a subgroup analysis including two groups with mild (QMGS $<10.5)$ and moderate to severe disease (QMGS > 10.5), only patients with mild disease and high decrement values $(\geq 10 \%)$ were more frequently treated with IVIG and PLEX (ORs 6.7 and 21.5, respectively), while high jitter values were associated with a higher frequency of mycophenolate treatment (Table 3).

The current study results show that MG patients with high decrement $(>10 \%)$ and jitter $(>100 \mu$ s) at baseline were considerably more likely to be treated during the follow-up period with immunomodulation, including IVIG and PLEX. These results are not surprising considering that more severely abnormal electrophysiology is associated with a more abnormal clinical status ${ }^{2,9}$ and a higher relapse rate. ${ }^{3}$ While IVIG or PLEX treatment is not infrequent in patients with a moderate to severe disease, it is rarely used in patients with mild disease, unless clinical deterioration occurs. However, there are currently no validated biomarkers allowing prediction of clinical deterioration in MG patients. Our study results show that high decrement at baseline in patients with mild disease is associated with a higher risk for future IVIG or PLEX treatment, even after adjusting for clinical severity (after splitting patients into two groups based on severity and adjusting OR for QMGS), reflecting potentially an independent prognostic role for decrement. Consequently, patients with mild disease and high decrement might benefit from more counselling regarding identifying and managing increasing symptoms earlier, and perhaps more frequent follow-up to detect clinical deterioration, although this assumption needs to be validated in prospective studies. Furthermore, although both patients with high jitter and decrement had more frequent bulbar and respiratory symptoms as baseline visit, only patients with high decrement continued to present more frequent bulbar and respiratory symptoms at the last follow-up visit, further supporting the utility of decrement as a robust prognostic tool. The absence of similar associations with high jitter might imply a need for a different cut-off value, or alternatively reflect the superiority of decrement recording for prognostic purposes.

Although patients with either high decrement or jitter had more abnormal clinical features at baseline, all showed a trend for improvement at the last follow-up visit, most likely reflecting treatment efficacy.

The current study has several limitations. It was designed as a retrospective study, and therefore the treating physician was not blinded to electrophysiological findings. However, as treatment decisions in our center are based on clinical grounds, it is unlikely that baseline electrophysiological findings had a significant impact on the decision to initiate immunomodulatory treatments such as IVIG or PLEX in later visits. Furthermore, we did not include a sufficient number of patients in order to explore differences in less frequent treatments, such as rituximab or cyclophosphamide, and had 12 patients with mild MG and decrement $\geq 10 \%$, and only 2 with decrement $\geq 20 \%$. In addition, we used electrophysiological data from a single cranial muscle (frontalis), and therefore cannot address the generalizability of the study results for other muscles. We also used previously defined cut-off values for jitter $(>100 \mu \mathrm{s})$ and decrement $(>10 \%){ }^{2}$ which may not be optimal for predicting response to different treatment regimens. Finally, our follow-up period was limited (less than 3 years), and therefore we cannot predict whether a similar trend would be demonstrated also in the 
Table 3: Univariable odds ratio (and 95\% confidence interval) of receiving indicated treatment in the presence of baseline jitter $\geq 100 \mu$ s or baseline decrement $\geq \mathbf{1 0 \%}$ and $20 \%$, for the whole cohort, and for patients with QMGS lower and greater than 10.5

\begin{tabular}{|c|c|c|c|c|c|c|}
\hline & Decrement $\geq 10 \%$ & $p$ & Decrement $\geq \mathbf{2 0 \%}$ & $p$ & Jitter $\geq 100 \mu \mathrm{s}$ & $p$ \\
\hline \multicolumn{7}{|c|}{ Total cohort $(n=87)$} \\
\hline$n$ (high/low) & $23 / 50$ & & $10 / 75$ & & $39 / 48$ & \\
\hline Pyridostigmine & $2.3(0.3-19.2)$ & 0.68 & $1.2(0.1-10.9)$ & 0.85 & $1.5(0.4-5.5)$ & 0.55 \\
\hline Azathioprine & $1.2(0.4-3.4)$ & 0.79 & $1.8(0.5-6.9)$ & 0.39 & $1.5(0.6-3.4)$ & 0.37 \\
\hline Mycophenolate & $2.8(0.9-8.8)$ & 0.07 & $2.1(0.5-8.3)$ & 0.29 & $1.7(0.7-4.3)$ & 0.28 \\
\hline Prednisone & $2.2(0.6-8.4)$ & 0.37 & $1.0(0.2-4.4)$ & 0.97 & $1.8(0.7-4.7)$ & 0.21 \\
\hline IVIG & $3.7(1.2-11.9)$ & 0.023 & $4.4(1.1-17.1)$ & 0.035 & $2.9(1-8.3)$ & 0.039 \\
\hline PLEX & $7.7(2-30)$ & 0.001 & $6.5(1.5-28.6)$ & 0.014 & $3.9(1-15.7)$ & 0.047 \\
\hline
\end{tabular}

Patients with QMGS $<10.5(n=55)$

\begin{tabular}{|c|c|c|c|c|c|c|}
\hline$n$ (high/low) & $12 / 42$ & & $2 / 52$ & & $23 / 32$ & \\
\hline Pyridostigmine & b & - & $\mathrm{b}$ & - & $1.8(0.3-11)$ & 0.51 \\
\hline Azathioprine & $2(0.3-11.5)$ & 0.46 & $\mathrm{~b}$ & - & $1.9(0.6-6.5)$ & 0.28 \\
\hline Mycophenolate & $13.9(1.9-102.7)$ & 0.01 & $4.1(0.2-73.1)$ & 0.34 & $5.9(1.3-27.4)$ & 0.022 \\
\hline Prednisone & $3(0.3-27.7)$ & 0.34 & $0.5(0.03-8.8)$ & 0.65 & $1.8(0.5-6.2)$ & 0.33 \\
\hline IVIG & $6.7(1.1-42.2)$ & 0.043 & $5.3(0.3-95)$ & 0.026 & $3.5(0.7-17)$ & 0.12 \\
\hline PLEX & $21.5(1.5-303.7)$ & 0.023 & $24.6(1-588)$ & 0.048 & $\mathrm{a}$ & 0.95 \\
\hline
\end{tabular}

Patients with QMGS > $10.5(n=18)$

\begin{tabular}{|c|c|c|c|c|c|c|}
\hline$n$ (high/low) & $8 / 11$ & & $7 / 11$ & & $10 / 8$ & \\
\hline Pyridostigmine & $\mathrm{b}$ & & $\mathrm{b}$ & & $\mathrm{b}$ & \\
\hline Azathioprine & $0.4(0.1-2.9)$ & 0.36 & $0.3(0.04-2.1)$ & 0.21 & $2.3(0.3-16.2)$ & 0.39 \\
\hline Mycophenolate & $2.5(0.3-22.5)$ & 0.40 & $3.4(0.4-29.4)$ & 0.26 & $1.3(0.2-10.5)$ & 0.82 \\
\hline Prednisone & $2.5(0.2-32.5)$ & 0.48 & $2.3(0.2-29.9)$ & 0.54 & $5.8(0.4-79.8)$ & 0.19 \\
\hline IVIG & $0.8(0.1-8)$ & 0.85 & $1.8(0.2-15.8)$ & 0.60 & $1.9(0.2-16.6)$ & 0.58 \\
\hline PLEX & $0.9(0.1-8.1)$ & 0.89 & $0.5(0.1-5.1)$ & 0.57 & $1.9(0.2-16.1)$ & 0.57 \\
\hline
\end{tabular}

QMGS=quantitative myasthenia gravis score; IVIG=intravenous immunoglobulins; PLEX=plasma exchange.

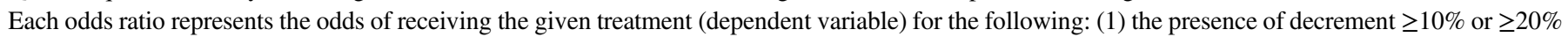

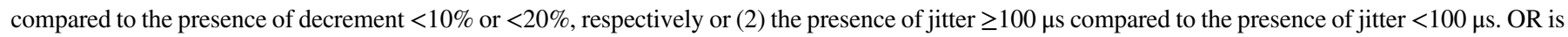
adjusted for QMGS. QMGS was not documented in 14 patients.

${ }^{\mathrm{a}}$ All patients with jitter $>100$ and QMGS $<10.5$ were treated with PLEX.

${ }^{\mathrm{b}}$ Could not be calculated due to low numbers.

Significant $p$-values $(<0.05)$ are shown in bold.

longer term. In conclusion, in patients with mild MG, more severely abnormal electrophysiological findings may be associated with more frequent exposure to more aggressive treatment regimens.

\section{CONFLict OF INTEREST}

Vera Bril has been a consultant for Bionevia, CSL, Dainnipon Sumitomo, Eisai, Grifols, Lilly, and Pfizer, and has received research support from Bionevia, CSL, Grifols, Takeda (Shire), Octapharma, Alexion, Argenx, Alynlam, UCB, Akcea, Pfizer, and Powell-Mansfield, outside the submitted work. Alon Abraham and Leif E. Lovblom have no conflicts of interest to declare.

\section{Statement of Authorship}

AA: study concept and design, analysis and interpretation of data. data.
VB: study concept and design, analysis and interpretation of data, study supervision, critical revision of manuscript for intellectual content.

\section{REFERENCES}

1. Sanders DB. Clinical impact of single-fiber electromyography. Muscle Nervei. 2002;999:S15-S20.

2. Abraham A, Breiner A, Barnett C, et al. Electrophysiological testing is correlated with myasthenia gravis severity. Muscle Nerve. 2017;56:445-448.

3. Baruca M, Leonardis L, Podnar S, et al. Single fiber EMG as a prognostic tool in myasthenia gravis. Muscle Nerve. 2016;54:1034-1040.

4. Limburg PC, The TH, Hummel-Tappel E, Oosterhuis HJ. Antiacetylcholine receptor antibodies in myasthenia gravis. Part 1. Relation to clinical parameters in 250 patients. J Neurol Sci. 1983;58:357-370.

5. Nations S, Wolfe GI, Mcintire D, Herbelin L, Barohn RJ, Bryan WW. Reliability testing of the quantitative myasthenia gravis scorea. Ann N Y Acad Sci. 2006;841:769-772. 
6. Abraham A, Alabdali M, Alsulaiman A, et al. Repetitive nerve stimulation cutoff values for the diagnosis of myasthenia gravis. Muscle Nerve. 2017;55:166-170.

7. Barnett C, Bril V, Kapral M, Kulkarni A, Davis AM. Development and validation of the myasthenia gravis impairment index. Neurology 2016;87:879-886.
8. Zinman L, Baryshnik D, Bril V. Surrogate therapeutic outcome measures in patients with myasthenia gravis. Muscle Nerve. 2008;37:172-176.

9. Barnett C, Katzberg H, Nabavi M, Bril V. The quantitative myasthenia gravis score: comparison with clinical, electrophysiological, and laboratory markers. J Clin Neuromuscul Dis. 2012;13:201-205. 